but evidence of direct glycinergic inhibition of the external urethral sphincter in Onuf's nucleus may be responsible for urinary retention.

\section{NeUrology (STROKe)}

\section{P.055}

\section{Distinguishable distribution of cerebral artery stenoses: ultrasonographic evidence from a northeast Chinese cohort}

H Liu (Changchun) X Wu (Changchun) Y Xing (Changchun) K Liu (Changchun) H Zhang (Changchun)*

doi: $10.1017 /$ cjn. 2015.165

Background: Cerebral artery stenosis is an important risk factor for ischemic strokes. This study aims to explore intracranial and extracranial artery stenosis in a large northeast Chinese cohort. Methods: We recruited 14793 outpatients and hospitalized patients to identify cerebral artery stenosis. Artery stenosis screening was done with transcranial Doppler (TCD) for intracranial arteries and carotid duplex sonography for extracranial arteries. Results: More intracranial than extracranial artery stenoses were identified (4255 versus 2809 , i.e. $28.8 \%$ versus $19.0 \%, \mathrm{P}<0.05$ ). Similarly, mere intracranial stenosis was significantly more common than extracranial artery stenosis in this population (2632 versus 1186 , i.e. $17.8 \%$ versus $8 \%$, $\mathrm{P}<0.05)$. Among all identified intracranial arteries stenoses, the proportion of middle cerebral artery (MCA) stenosis was the highest. More intracranial than extracranial artery stenoses was seen within each age group, and rates of both increased with age. Intracranial and extracranial artery stenosis was more frequently identified in males than females. Conclusions: Incidence of cerebral artery stenosis in the population increases with age. Intracranial artery stenosis is more common than extracranial artery stenosis and the MCA stenosis accounted for the highest proportion, within each age group. More males suffer from intracranial or extracranial artery stenosis than females.

\section{P.056}

Noninvasive assessment of ischemic penumbra by using MR-SWI during the acute phase of cerebral infarction: a comparison to $\mathrm{PWI}$

$S$ Luo (Changchun) $X$ Wu (Changchun) F Deng (Changchun) $Y$ Zhang (Changchun) J Miao (Changchun) Y Chen (Changchun) L Wang (Changchun) J Feng (Changchun) H Zhang (Changchun)*

doi: $10.1017 / \operatorname{cjn} .2015 .166$

Background: Assessment of ischemic penumbra during the acute stage of cerebral infarction is crucial for a decision to initiate thrombolytic therapy and for predicting stroke evolution. Although controversial as a perfect equivalence to penumbra, perfusion weighted imaging (PWI)-diffusion weighted imaging (DWI) mismatch may predict the response to thrombolysis. Due to the reliance on contrast agents in PWI, noninvasive alternatives remain an unmet need. Methods: We herein investigate the potentials of SWI as an alternative to PWI in defining ischemic penumbra and in predicting stroke outcome. A multimodal magnetic resonance imaging work-up which includes conventional magnetic resonance imaging sequences (T1WI, T2WI and FLAIR), DWI, PWI and SWI was performed. The Alberta Stroke Programme Early CT Score (ASPECTS) was used to evaluate the changes in DWI, SWI and PWI. Results: The mismatch of SWI-DWI was comparable with that of PWI-DWI ( $p>0.05)$. Furthermore, the grade of prominent vein and the cerebral blood volume in the ipsilateral brain tissue were positively correlated. Conclusions: SWI can be used as a noninvasive alternative to identify occlusive arteries and to evaluate the ischemic penumbra. The susceptibility vein sign may represent thrombosis in arteries whereby being helpful to identify responsible blood vessels in ischemic stroke.

\section{P.057}

Different strokes for different folks: epidemiology of cerebrovascular diseases amongst Chinese-Canadians residing in Toronto

\author{
JY Chu (Toronto)* JK Chu (Atlanta) DK Chu (Hamilton) S Lam (To- \\ ronto)
}

doi: $10.1017 / \operatorname{cjn} .2015 .167$

Introduction: It has been recognized in the past few decades that different ethnic groups living in Canada may have different stroke epidemiology. This presentation is focused on the stroke patterns of Chinese-Canadians living in the Toronto area. Methods: Two retrospective case-controlled studies were carried out between 1990- 2000 to study the stroke characteristics of Chinese-Canadians living in Toronto. Statistical analysis was carried out by the Institute of Clinical Evaluative Sciences. A further retrospective study was also carried out in 2011 to look at the relationship between stroke and diabetes mellitus amongst this population. Results: Chinese-Canadians were found to have $1 / 6$ the prevalence of extracranial vascular stenosis. They have a higher frequency of intracranial vascular disease which may be due to the higher frequency of hypertension and diabetes mellitus. Higher incidence of intracranial hemorrhage was found compared to Caucasian controls which may be due to the lack of awareness and optimal treatment of their hypertension. Details of the results of these three studies will be presented. Conclusions: This is the first long term retrospective study of the stroke patterns and epidemiology for Chinese-Canadians residing in Toronto. Further prospective population-based study will be vital to study the important interactions between genetics and environment in the pathogenesis of different strokes for different folks.

\section{P.058}

Bilateral thalamic infarction due to artery of percheron occlusion with corresponding CT perfusion abnormalities

\author{
A MacLellan (Toronto)* K Boyle (Toronto)
}

doi: 10.1017/cjn.2015.168

Background: The Artery of Percheron (AOP) is a rare anatomic variant that supplies the bilateral medial thalami from a common origin; occlusion results in a characteristic pattern of bilateral thalamic infarction seen on neuroimaging. To date, we have not identified any cases in the literature describing corresponding ischemic changes seen on hyperacute CT Perfusion imaging. We aimed to characterize perfusion abnormalities seen in AOP occlusion by describing a case 